\title{
Heart Rate Variability-A Review
}

\author{
Vyasaraj $\mathrm{T}^{1}$, Veena $\mathrm{N}$ Hegde 2 \\ ${ }^{1}$ Dept. of ECE, BMSCE, Bengaluru, Assistant Professor Dept. of ECE, BNMIT, Bengaluru, VTU, \\ Belagavi, Karnataka India, \\ 2 Dept. of E\&I Engineering, BMS College of Engineering, Bengaluru, Karnataka India. \\ vyasaraj.t@gmail.com ${ }^{2}{ }^{2}$ veenahegdebms.intn@bmsce.ac.in
}

\begin{abstract}
Information about the health can be obtained by measuring various physiological parameters such as Heart Rate, Heart Rate Variability (HRV), Nerve conduction, brain activity, blood oxygen saturation level etc. The useful information resulted from the systematic analysis of these physical parameters are helpful for clinicians to make better decisions. HRV reflects the state of the Autonomic Nervous System (ANS) defined as the variance in the time between successive heartbeats expressed in milliseconds. The various factors that affects HRV are diet, nutrition, age, alcohol, gender, cardiac rhythm, sleep habits, genetics etc. The analysis of HRV is helpful in stress assessment and also in identifying the diseases at the early stage. This paper discusses the fundamentals of HRV, analysis of HRV, and the role of HRV in stress detection.
\end{abstract}

\section{Keywords- Autonomic Nervous System (ANS), Heart Rate Variability (HRV)}

\section{Introduction}

The two physiological parameters that have received excellent attention recently are Heart rate (HR) and Heart rate variability (HRV). The number of heart beats per minute is referred as Heart rate. The low value of heart rate indicates body is at rest, where as a under scenarios corresponding to exercise or exertion heart rate is normally high. The another physiological marker Heart rate Variability (HRV) is the variation in time between each heart beats which is expressed in milliseconds. Basically, a lower value of HRV is associated with the body which is under stress because of exercise activities, any physiological events etc, on the other side a higher value of HRV is associated with the body having a strong ability to tolerate stress and higher value of HRV at rest indicates better health. Electrocardiogram (ECG) and Photoplethysmography (PPG) are the two most preferred techniques used for obtaining the HRV. Nervous system majorly consists of two divisions namely Central Nervous System (CNS) and Peripheral Nervous System (PNS). The brain, medulla oblongata and spinal cord constitutes CNS, whereas the nerves that branch off from the spinal cord and extends to all parts of the body constitutes PNS. Peripheral Nervous System is subdivided into Somatic Nervous System (SNS) and Autonomic Nervous System (ANS). Somatic Nervous System is responsible for transmission of sensory and motor signals to and from the central nervous system. The Autonomic Nervous System (ANS) is responsible for the regulation of cardiac activity and some of the body processes, such as blood pressure, breathing rate, body temperature etc. As the Heart rate and heart rhythm are majorly under the control of ANS, the analysis of HRV becomes a good indicator for assessing the overall cardiac health and also the state of the ANS. Therefore HRV is considered as a noninvasive marker of ANS and is the key component in analyzing the various diseases and ANS dysfunction. Sympathetic Nervous System (SNS) and Parasympathetic Nervous System (PNS) are the two major subdivisions of ANS. Sympathetic nervous effects include increase in HR, dilation of pupil, faster breathing dry mouth, constriction of blood vessels except the coronary vessels etc. Para sympathetic nervous system re-establishes normal conditions after the 
abnormal condition is over. Overall two subdivisions work together such that body responds to different conditions appropriately.

\section{Analysis of Heart Rate Variability}

The ECG signal corresponding to each heart beat contains quasi-periodic sequences namely P wave, QRS complex and T- wave as shown in figure 1. In this sequence QRS complex has highest amplitude and it helps in determining the time difference between the two consecutive heart beats referred as RR interval.

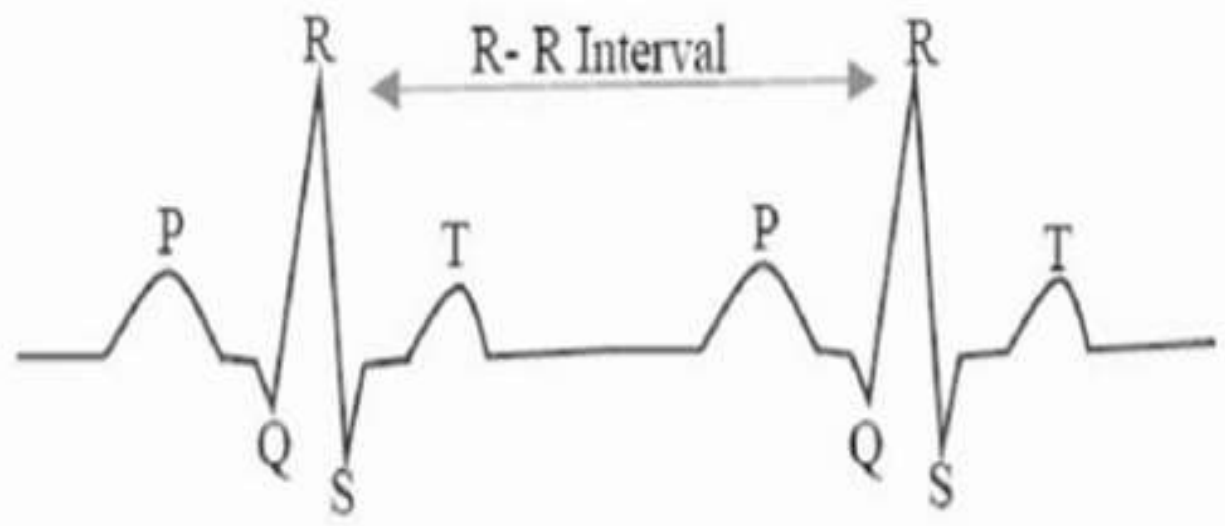

Fig 1: The normal electrocardiogram with components

The following steps are involved in the analysis of HRV

- Collecting ECG data

- Filtering (Removal of Noise present in HRV)

- Detecting R Peaks

- Generation heart rate variability signal

- Extracting the different features

The flowchart of the analysis of HRV is as shown in figure 2.

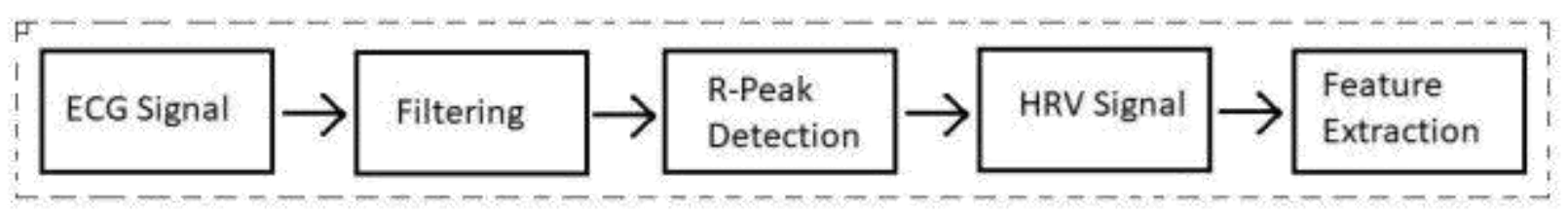

Fig 2: HRV analysis flowchart

ECG data required for analyzing the HRV signal can be obtained from the internet by using the databases of ECG and also from the people on voluntary basis. ECG data which is in analog format need to be digitized by suitable sampling frequency. Due to the muscle contraction or movement of patients during the recording of ECG some noise might be generated in the ECG signal which should be minimized by using a suitable filter. After de-noising the ECG signal, the next part is to calculate R-peaks detection.

The R peaks are identified by observing the maximum upward deflection in the QRS complex and the RR interval which is referred to as HRV can be obtained by calculating the time interval between the two consecutive heart beats. If $\mathrm{R}$ peaks identified at samples $\mathrm{k}=1,2,3, \ldots \ldots \ldots \mathrm{N}$, then the RR length is given by $\mathrm{R}_{\mathrm{k}+1}-\mathrm{R}_{\mathrm{k}}$. 
There are various techniques involved in QRS detection. Pan and Tompkins algorithm for QRS detection is emerged as one of the best methods. The different steps involved in Pan and Tompkins algorithm are filtering, differentiation, squaring, and moving integrator.

Once the HRV signal is obtained it is analyzed for obtaining the time domain, frequency domain and non-linear domain features. The time domain and frequency domain features are together known as linear features. Time domain features are calculated by taking time as a reference and it indicates changes in heart rate w.r.t time, normally performed on long term recordings. The features which are used for analyzing the HRV in time domain are; SDNN measured in milliseconds is the standard deviation of all RR intervals, SDANN measured in milliseconds is the standard deviation of the averages of RR intervals in all 5-minute segments of the entire recording, SD measured in milliseconds is the standard deviation of differences between adjacent RR intervals, RMSSD measured in milliseconds is the square root of the mean of the sum of the squares of differences between adjacent RR interval, PNN50 is the percent of difference between adjacent RR intervals that are greater than $50 \mathrm{~ms}$.

The power spectral density of RR intervals are calculated using the Fourier transform to obtain the frequency domain features. The power spectral density contains power spectrum of very low, low and high frequency components in the range 0 to $0.04 \mathrm{~Hz}, 0.04$ to $0.4 \mathrm{~Hz}$, and greater than $0.4 \mathrm{HZ}$ respectively. The activities of SNS and PNS are indicated by low frequency component and high frequency components respectively. The various parameters which can be considered for frequency domain analysis are; total power which is measured $\mathrm{ms}^{2}$ indicates the Variance of all RR intervals having a frequency less than $0.4 \mathrm{~Hz}$,

Ultra-low frequency which is less than $0.003 \mathrm{~Hz}$, Very low frequency in the range $0.0003 \mathrm{~Hz}-0.04 \mathrm{~Hz}$, low frequency power in the range $0.04 \mathrm{~Hz}-0.15 \mathrm{~Hz}$, high frequency power in the range $0.15 \mathrm{~Hz}-0.4 \mathrm{~Hz}$ and the ratio of low frequency to high frequency power. Another method is used for analyzing HRV signal is non-linear analysis, the methods such as Poincare Plot, Correlation Dimension Analysis can be used for analyzing the HRV which also gives very useful information.

\section{HRV and Stress}

ECG data are generally very noisy, so filtering techniques are used to de-noise the ECG data, then Rpeaks are detected. Time Domain, Frequency Domain, Wavelet Domain and Non-Linear Techniques are the different methods used for analyzing the HRV. Low value of HRV is indicator of adverse events in healthy individuals, whereas in patients with diseases it reflects the role of ANS. The important clinical information which are helpful clinicians can be obtained from the early measurements of HRV during Acute Myocardial Infarction (AMI). Basically HRV decreases under the emotional stress or physical stress situations and it increases with the rest [1-5].

Pulse rate, temperature of the skin and level of skin conductance are the three parameters which are used for stress management as they are the marker of sympathetic activation in response to stress reaction. Using Internet of Things a low power cost effective wearable band which functions based on skin conductance is designed to detect metal stress. Sweat glands are activated by SNS when a person is under stress, and the sweat produced from the sweat glands increases the conductance of the skin by reducing the skin resistance. The inner side of the wearable band contains two electrodes which measures the skin conductance and by using different algorithms to analyze the output voltage, the stress condition of the subject is predicted [6]. 
The ECG data of 30 students are acquired during their examination time in order to analyze the mental stress. The obtained HRV signal from the processing of ECG signal are analyzed to obtain time, frequency and geometrical features. The lower value of the time domain feature RMSSD corresponds to stress of a person, whereas the frequency domain feature of low frequency to high frequency ratio with a higher value indicates the stress. The Poincare plot analysis is used as the geometrical feature and the standard deviations for both short term and long term recordings are calculated and in both the cases the lower values of standard deviation is observed under stress [7].

The stress levels of driver is investigated on a set of ECG data taken from the automobile driver database including the data from the sixteen healthy individual drivers on a voluntary process. The obtained ECG signal is processed for obtaining HRV signal and HRV is analyzed to measure time, frequency, timefrequency and non-linear features to build a vector of features as a input to the classification stage. Different classification techniques such as Support Vector Machine (SVM) with radial basis function $(\mathrm{RBF})$, as K Nearest Neighbor (KNN) and the radial basis function (RBF) are used to classify the stress state or normal state of the person [8]. The changes in the morphology of ECG beats referred as morphological variability and HRV are obtained by processing the 16 different ECG signals taken from the database. The various time domain and frequency domain metrics of HRV are analyzed for classifying the low, medium and high stress states and frequency domain metrics of morphologic variability are used for classifying the low, medium and high stress states. The results depicts that morphologic variability analysis is better in comparison with the HRV analysis [9].

Unobtrusive wearable sensors are designed to detect mental stress. The nonlinear technique named as Principal Dynamic Modes (PDM) is used to analyze the HRV obtained from Heart rate Monitors (HRM) in order to estimate the state of ANS. The data used for the experiment is collected from three subjects under two evoked mental stress and two induced relaxation conditions. The obtained heart period signals from HRM are processed to identify $\mathrm{R}$ peaks using peak detection algorithm and RR intervals are obtained. The low frequency power and high frequency power from PSD and features of PDM are extracted for classification using $\mathrm{K}$ nearest neighbor. The results obtained ensures that the PDM is more stable than the PSD [10].

Ultra-short term HRV analysis technique is used to measure the HRV for classifying the state of the mental stress and state of normal for the subjects under observation. In this experiment 60 segments of $\mathrm{RR}$ interval recorded from six subjects are used for analysis purpose. The results ensures that the time domain features mean of RR intervals, mean of heart rates and frequency domain features namely; normalized low frequency, difference between the normalized low frequency and normalized high frequency, Symphatovagal balance index which is the ratio of low frequency to high frequency are the effective measures for identifying the mental stress [11].

A $65 \mathrm{~nm}$ stress detector is implemented to distinguish stress among three stress states namely, low, medium and high. The ECG data of seventeen drivers experiencing the three states of stress are obtained from the physio net data base. The ECG data is processed using Pan Tompkins algorithm to obtain the QRS complex and thus HRV signal. The HRV data is analyzed for obtaining the various parameters in time domain and frequency domain. In the classification stage time domain parameters such as heart rate, root mea square of RR intervals and mRR are used to classify between the stress or normal states. The two stage support vector machine classifier is used which further improves the overall accuracy of the system by classifying the stress state again into either medium or high stress [12]. Relationship between subjective sleep quality assessment and heart rate variability indices are examined. HRV associated with cardiac vagal function were correlated with subjective sleep quality [13]. The influence of different 
physiological factors on HRV in predicting the cardiovascular diseases is discussed. To analyze HRV, ECG data is obtained from MIT database which contains data from both disorder and healthy group. LabVIEW software is used for processing the ECG and obtaining the various time and frequency domain features. The results obtained ensures the difference in time and frequency domain parameters in normal and healthy patients. The results also ensures that the higher value of low frequency components found in males indicating the dominance of sympathetic nervous system in males and higher value of high frequency feature observed in females indicating the parasympathetic dominance [14]. HRV analysis gives a valuable information as it reflects the functioning of the ANS, and hence it can be used as implication in various medical diseases. The neurobiology of psychiatric disorders can be well understood by investigating the Autonomic Nervous System as ANS dysregulation may be due to the some psychiatric disorders [15].

\section{CONCLUSION}

There are several works have been done in analyzing the HRV signal by using time and frequency domain features. The analysis of HRV gives a valuable information for clinicians for the better diagnosis of patients. In the literature, analysis of stress level in different scenarios have been analyzed, and there is scope for improving the reliability of the results by considering a larger data sets. The HRV analysis can be further extended to analyze the different psychiatric disorders by investigating the ANS dysregulation as the obtained features from HRV analysis are helpful in knowing the symptoms related to psychiatric disorders which leads to ANS disturbances.

\section{REFERENCES:}

[1] Hoang ChuDuc, Kien NguyenPhan, Dung NguyenViet "A Review of Heart Rate Variability and its Applications" APCBEE Procedia 7 (2013) 80-85. (Journal)

[2] Nasim Karim et al. "Heart Rate Variability- A review" Journal of Basic and Applied Sciences Vol. 7, No. 1, 71-77, 2011. (Journal)

[3] Mohamed Faisal Lutfi "Review article: Heart rate variability" Sudan JMS Vol. 6, No.1. Mar 2011. (Journal)

[4] Fuan Sztajzel "Heart rate variability: a noninvasive electrocardiographic method to measure the autonomic nervous system" SWISS MED WKLY 2004;134:514-522. (Journal)

[5] Desh Deepak Gautam, V.K.Giri “Analysis of HRV Signal for Disease Diagnosis" $201611^{\text {th }}$ International conference on Industrial and Information systems. (Conference)

[6] Muhammad Zubair, Changwoo Yoon, Hyunyoung Kim, Jisu Kim, Jinsul Kim "Smart Wearable Band for Stress Detection” IEEE 2015. (Journal)

[7] Ramyashri Ramteke, V. R. Thool "Stress Detection of Students at Academic Level from Heart Rate Variability" International Conference on Energy, Communication, Data Analytics and Soft Computing (ICECDS-2017). 2017 IEEE. (Conference)

[8] Nermine Munla, Mohamad Khalil, Ahmad Shahin and Azzam Mourad "Driver Stress Level Detection Using HRV Analysis" 2015 International Conference on Advances in Biomedical Engineering (ICABME). 2015 IEEE. (Conference)

[9] Hariton Costin, Cristian Rotariu, Alexandru Pasarica "Mental Stress Detection using Heart Rate Variability and Morphologic Variability of ECG Signals" 2012 International Conference and Exposition on Electrical and Power Engineering (EPE 2012), 25-27 October, Iasi, Romania. 2012 IEEE. (Conference) 
[10] Jongyoon Choi and Ricardo Gutierrez-Osuna "Using Heart Rate Monitors to Detect Mental Stress" 2009 Body Sensor Networks. 2009 IEEE. (Journal)

[11] Sansanee Boonnithi, Sukanya Phongsuphap "Comparison of Heart Rate Variability Measures for Mental Stress Detection” Computing in Cardiology 2011;38:85-88, ISSN 0276-6574. (Journal)

[12] Huda Goian, Aamna Alali, Temesghen Habte, and Hani Saleh "A 65nm ASIC Design for Measuring Mental Stress from The Heart Rate Variations" 2017 IEEE. (Journal)

[13] E Yuda, Y Yoshida, J Hayano "Relationship between subjective assessment of sleep quality and heart rate variability during sleep" 2018 IEEE International Conference on Consumer Electronics-Taiwan (ICCE-TW). (Conference)

[14] Hemant Kasturiwal, Sujata N. Kale "Influence of physiological factors on Heart Rate Variability in predicting cardiovascular diseases" Proceedings of the 2nd International Conference on Communication and Electronics Systems (ICCES 2017), ISBN:978-1-5090-5013-0. (Conference)

[15] Albert C. Yang, M.D, Chen-Jee Hong, M.D., Shih-Jen Tsai, M.D "Heart Rate Variability in Psychiatric Disorders" Taiwanese Journal of Psychiatry (Taipei) Vol. 24 No. 2 2010. (Journal)

[16] Aleš Procházka, Hana Charvátová, Saeed Vaseghi, And Old rich Vyšata, "Machine Learning in Rehabilitation Assessment For Thermal And Heart Rate Data Processing" IEEE Transactions On Neural Systems And Rehabilitation Engineering, Vol. 26, No. 6, June 2018. (Journal)

[17] Rollin McCraty, "Heart Rate Variability: New Perspectives on Physiological Mechanisms, Assessment of Self-regulatory Capacity, and Health Risk" Global Adv Health Med. 2015;4(1):46-61. DOI: 10.7453/gahmj.2014.073. (Journal)

[18] Wan-Hua Lin, Dan Wu, Chunyue Li, Heye Zhang, and Yuan-Ting Zhang, "Comparison of Heart Rate Variability from PPG with That from ECG" The International Conference on Health Informatics, IFMBE Proceedings 42, 213 DOI: 10.1007/978-3-319-03005-0_54, () Springer International Publishing Switzerland 2014. (Conference)

[19] Vala Jeyhani, Shadi Mahdiani, Mikko Peltokangas and Antti Vehkaoja "Comparison of HRV Parameters Derived from Photoplethysmography and Electrocardiography Signals" 2015 37th Annual International Conference of the IEEE Engineering in Medicine and Biology Society (EMBC), DOI 10.1109/EMBC.2015.7319747. (Conference) 